\title{
Chitosan Improves the Biological Performance of Soy-Based Biomaterials
}

\author{
Tírcia C. Santos, Ph.D., ${ }^{1-3}$ Alexandra P. Marques, Ph.D., ${ }^{1,2}$ Simone S. Silva, Ph.D., ${ }^{1,2}$ \\ Joaquim M. Oliveira, Ph.D., ${ }^{1,2}$ João F. Mano, Ph.D., ${ }^{1,2}$ António G. Castro, Ph.D., ${ }^{4}$ \\ Martijn van Griensven, M.D., Ph.D., ${ }^{3}$ and Rui L. Reis, Ph.D.,
}

Soybean protein has been proposed for distinct applications within nutritional, pharmaceutical, and cosmetic industries among others. More recently, soy-based biomaterials have also demonstrated promising properties for biomedical applications. However, although many reports within other fields exist, the inflammatory/immunogenic potential of those materials is still poorly understood and therefore can hardly be controlled. On the contrary, chitosan (Cht) has been well explored in the biomedical field, either by itself or combined with synthetic or other natural-based polymers. Therefore, the combination of chitosan with soybean protein is foreseen as a suitable approach to control the biological behavior of soy-based biomaterials. Under this context this work was designed to try to understand the influence of chitosan in the host response elicited by soy-based biomaterials. Soybean protein isolate powder (SI-P) and Cht powder (Cht-P) were injected as suspension into the intraperitoneal cavity of rats. SI-P induced the recruitment of higher numbers of leukocytes compared to the Cht-P during the entire observation period. In this sense, SI-P elicited a considerable reaction from the host comparing to the Cht-P, which elicited leukocyte recruitment similar to the negative control. After subcutaneous implantation of the soybean and denatured membranes, (SI-M and dSI-M) a severe host inflammatory reaction was observed. Conversely, Cht/soy-based membranes (Cht/soy-based membranes) showed the induction of a normal host response after subcutaneous implantation in rats, which allowed concluding that the addition of chitosan to the soy-based membranes improved their in vivo performance. Thus, the presented results assert the improvement of the host response, considering inflammatory cells recruitment, and overall inflammatory reaction, when chitosan is combined to soybean. Together with previous results that reported their promising physicochemical characteristics and their inability to activate human polymorphonuclear neutrophils in vitro, the herein presented conclusions reinforce the usefulness of the Cht/ soy-based membranes and justify the pursue for a specific application within the biomedical field.

\section{Introduction}

$S_{\mathrm{i}}^{\text {o }}$ OYBEAN PROTEIN BIOMATERIALs have demonstrated quite interesting properties for bone regeneration purposes ${ }^{1-5}$. nonetheless, few studies ${ }^{6-8}$ have in fact investigated their suitability within the field. Santin et al. ${ }^{9}$ showed that soybean-based biomaterials promote osteoblast-like cells' differentiation in vitro without inducing activation of human macrophages (MØ). ${ }^{9}$ However, additional studies are required to further elucidate the potential of these materials in the biomedical field. Moreover, despite the wide use of soy products in nutritional, pharmaceutical, cosmetic, and many other industries, ${ }^{10}$ numerous allergic reactions, from skin, gastrointestinal and respiratory tract up to anaphylaxis, ${ }^{10,11}$ have been attributed to the soy Gly $\mathrm{m} 4$ protein, homologous of the major birch pollen allergen, Bet $\mathrm{v} 1 .^{11}$

In contrast to soybean protein, chitosan has been extensively proposed in the biomedical arena for bone-, ${ }^{12-16}$ cartilage-, $13,17,18$ and skin-related ${ }^{13,19,20}$ applications. The promotion of osteoblast proliferation and activity characterized by upregulated expression of bone-related proteins and mineral-rich matrix deposition, directed by chitosan-based structures, has been well demonstrated. ${ }^{15,16}$ Concerning cartilage-related applications, the structural similarity of

The present work was developed at the 3B's Research Group, Portugal and at the Ludwig Boltzmann Institute for Experimental and Clinical Traumatology, Austria.

${ }^{13 B}$ 's Research Group-Biomaterials, Biodegradables, and Biomimetics, University of Minho, Headquarters of the European Institute of Excellence on Tissue Engineering and Regenerative Medicine, Guimarães, Portugal.

${ }^{2}$ IBB-Institute for Biotechnology and Bioengineering, PT Associated Laboratory, Guimarães, Portugal.

${ }^{3}$ Ludwig Boltzmann Institute for Experimental and Clinical Traumatology, Austrian Cluster for Tissue Regeneration, Vienna, Austria.

${ }^{4}$ Life and Health Sciences Research Institute, School of Health Sciences, University of Minho, Braga, Portugal. 
chitosan with various glycosaminoglycans found in articular cartilage has burst the investigations. Besides playing a role in modulating chondrocyte morphology, differentiation, and function, ${ }^{18}$ chitosan was shown to act on the growth of epiphyseal cartilage and wound healing of articular cartilage. ${ }^{17}$ The successful role of chitosan in the skin-woundhealing mechanisms has been confirmed. ${ }^{19-21}$ Although it was observed that chitosan does not directly accelerate extracellular matrix production by fibroblast-like cells, ${ }^{22}$ it was proven that chitosan-based materials have the capacity to promote the production of growth factors, such as transforming growth factors- $\beta 1$ and platelet-derived growth factor by $\mathrm{MØ}^{21}$ which, in turn, induce and/or enhance extracellular matrix production. ${ }^{20,21}$ Moreover, these materials accelerate the infiltration of polymorphonuclear neutrophils (PMNs) at the early stage of wound healing that is followed by the production of collagen by fibroblasts. ${ }^{19}$

Despite all the promising results in the use of Cht for the biomedical field, studies from different groups ${ }^{21,23,24}$ have shown controversial results after implanting Cht-based materials. An adverse inflammatory response showing extensive $\mathrm{MØ} \mathrm{activation} \mathrm{after} \mathrm{subcutaneous} \mathrm{implantation} \mathrm{of} \mathrm{either}$ lyophilized chitosan ${ }^{21}$ or collagen-chitosan-hydroxyapatite hydrogels in rats ${ }^{23}$ was detected. Conversely, other authors showed that chitosan hydrogels induce mild acute and chronic inflammatory responses, identical to the typical wound-healing cascade, after subcutaneous ${ }^{25}$ and intraperitoneal (IP) implantations in rats. ${ }^{24}$

Nevertheless, the significance of chitosan within the biomedical field is unquestionable since the observed differences on the response of cells and tissues to the different chitosanbased materials may be attributed or influenced by the source of the raw material or by the shape of the biomaterial. Chitosan has also been one of the most used materials to improve the biological performance of other materials such as metals ${ }^{26-29}$ and other biodegradable polymers., ${ }^{76,30}$ Recent studies demonstrated that neonatal rat calvaria osteoblasts proliferate at higher rates on titanium surfaces coated with chitosan, ${ }^{26}$ which also promote better adhesion of osteoblast-like cells. ${ }^{27}$ In the same way, the hydrophobic surface of poly(L-lactide) matrices coated with chitosan displayed a different wettability and enhanced cell affinity. ${ }^{28}$ In a rabbit tibia defect model, Bumgardner and co-workers ${ }^{29}$ showed that the coating of titanium pins with chitosan induced minimal inflammatory response and a typical healing sequence of fibrous woven bone formation followed by the development of lamellar bone. This is indicative for the role of the chitosan-coatings in the osseointegration of orthopedic implants. ${ }^{29}$ When blended with synthetic polymers, such as polycaprolactone ${ }^{16}$ or poly (butylene succinate), ${ }^{30}$ chitosan have shown to exert a synergistic effect of on the blend. While the synthetic polyester promoted the adhesion of osteoblast-like cells, the presence of chitosan significantly enhanced their osteoblastic activity. ${ }^{16,30}$ Chitosan/soy-based membranes (Cht/Soy-M) have also demonstrated improved in vitro biological performance in comparison to unblended soy-based materials. ${ }^{7}$ Additionally, in vitro analysis of the potential of Cht/Soy-M to stimulate immune system cells showed that they did not elicit the activation of human PMNs freshly isolated from circulating blood. ${ }^{31}$ Despite the in vitro promising results, in vivo validation of the improved biological behavior of the soy-based materials after blended with Cht is needed, since the organism includes a very complex immune system. Therefore, the aim of this study was to test the influence of chitosan in the host response provoked by soy-based biomaterials. The response to the raw materials soybean protein isolate powder (SI-P) and chitosan powder (Cht-P) was assessed after injection in the intraperitoneal cavity of rats. The in vivo reaction to soybean protein isolate membrane (SI-M) and Cht/Soy-M was compared after subcutaneous implantation of the biomaterials. The results from the different models and the comparison of the performance of the SI- and the chitosan-blended membranes allowed to conclude about the effect of the chitosan over the in vivo behavior of soy-based membranes.

\section{Materials and Methods}

\section{Materials}

The tested materials were (1) SI-P, (2) Cht-P, (3) SI-M and (4) Cht/Soy-M. SI was provided by Loders Crocklaan BV (The Netherlands) and the reagent-grade Cht, with a deacetylation degree of $85 \%$ and viscosimetric molecular weight of about $700 \mathrm{KDa}$, by Sigma. Before the experiments, Cht was purified by re-precipitation method. ${ }^{32}$ The SI-Ms were prepared by solvent casting, according to a previously reported procedure. ${ }^{33}$ Briefly, SI was suspended in distilled water $(10 \%, \mathrm{w} / \mathrm{v})$ at room temperature under gentle stirring to avoid protein denaturation and, consequently, foam formation. Glycerol was added to this suspension ( $1 \mathrm{~g}$ per $5 \mathrm{~g}$ of $\mathrm{SI}$ ), which was then poured into molds, directly in the drying place. The molds were not moved until complete drying to assure that the insoluble part of SI was uniformly distributed. Drying was performed at room temperature and relative humidity. Alternatively, denatured SI membranes (dSI-M) were prepared by heating the referred SI suspensions at $100^{\circ} \mathrm{C}$ during $2 \mathrm{~h}$. After denaturation, the obtained viscous solution was cast as described above. Cht/Soy-M were produced by solvent casting method. ${ }^{8}$ A 4 wt $\%$ Cht solution was prepared by dissolving $\mathrm{Cht}$ in a $0.2 \mathrm{M}$ acetic acid solution. A $1 \mathrm{wt} \%$ soy suspension (water/glycerol $[10 \%$, $\mathrm{w} / \mathrm{v}]$ ) was also prepared and the $\mathrm{pH}$ adjusted to $8.0 \pm$ 0.3 with $1 \mathrm{M}$ sodium hydroxide solution. Previous reports showed that the addition of glycerol can accelerate the membrane formation process, ${ }^{34}$ while the alkaline conditions help the soy film formation by adding protein dispersion in film-forming solutions. ${ }^{35}$ The dispersion was heated in a water bath at $50^{\circ} \mathrm{C}$ for $30 \mathrm{~min}$. The blend was prepared by means of mixing the solutions $(75 / 25 \mathrm{wt} \%$ chitosan-soy protein) under constant agitation for $1 \mathrm{~h}$. After the chitosansoy protein solutions had been homogenized, they were cast into Petri dishes and dried at room temperature. To neutralize the acetic acid, the dried membranes were immersed in $0.1 \mathrm{M}$ sodium hydroxide for about $10 \mathrm{~min}$, and then washed with distilled water to remove all traces of alkali, followed by drying at room temperature.

\section{Animals}

Twenty-four male Wistar Han rats (Charles River) weighting 420-450 g were used for the subcutaneous implantation of SI-M and dSI-M, as well as for the IP injection of SI-P and Cht-P. Four male Sprague-Dawley rats (Himberg breeding Institute) weighting 320-470 g were used for the subcutaneous implantation of the Cht/Soy-M. Animal ex- 
perimentation was performed according to the standard operating procedures approved by the national authorities.

\section{Intraperitoneal injection of SI-P and Cht-P}

Phosphate-buffered saline (PBS; 0.01M; Sigma-Aldrich) suspensions of the powders with different concentrations $(0.1 \%$ and $1 \% \mathrm{SI}$, and $0.1 \%, 1 \%$, and $2 \% \mathrm{Cht})$ were injected into the IP cavity of the rats $(1 \mathrm{~mL}$ per animal). Three animals per concentration and per time period (16h, 3 days, 7 days, and 15 days) were used. One animal per each concentration and per time period was injected with sterile PBS as control. The animals had access to food and water ad libitum during the entire observation period.

\section{Subcutaneous implantation of membranes}

I. Subcutaneous implantation of the SI-M. For the implantation of the SI-M and the dSI-M, the Wistar Han rats were anesthetized by an IP injection of $2.5 \%$ pentobarbital (CEVA Santé Animale). The interscapular region was shaved and disinfected with $70 \%$ ethanol, a full-thickness skin longitudinal incision (about $1.5 \mathrm{~cm}$ ) was performed in each animal and one cranial-oriented subcutaneous pocket was created by blunt dissection. A membrane (12 $\mathrm{mm}$ diameter) was positioned into each pocket and the incision was carefully sutured. Three animals were used per each time period of implantation (3, 7, 15, and 30 days), and per type of membrane. For each time period of implantation, a control animal, with an empty subcutaneous pocket, was set. The animals were kept in single cages with food and water ad libitum during the whole experimentation period.

II. Subcutaneous implantation of the Cht/Soy-M. Male Sprague-Dawley rats were anesthetized before surgery by intramuscular injection of $90 \mathrm{mg} / \mathrm{kg}$ ketamine hydrochloride (Schoeller Chemie Produkte) and $5 \mathrm{mg} / \mathrm{kg}$ xylazine hydrochloride (Bayer AG). For the subcutaneous implantation of the Cht/Soy-M, two medial full-thickness skin incisions were performed on the dorsum of the rats. Two craniolateraloriented pockets per each incision one to the left and one to the right were subcutaneously created by blunt dissection, and the membranes were inserted into these pockets (four membranes/animal). The animals were kept in single cages with food and water ad libitum during the whole experimentation period.

\section{Cytological preparations}

The kinetics of the inflammatory reaction induced by the injected suspensions (made from the powders) was assessed by analysis of the peritoneal exudate. The animals were anesthetized with a subcutaneous injection of $2.5 \%$ pentobarbital and sacrificed with an intracardial overdose of anesthetic. The abdominal area of each animal was disinfected with $70 \%$ ethanol, and an incision in the abdominal skin and the linea alba was made to expose the external abdominal wall as most as possible to the subsequent lavage. Thirty-five milliliters of sterile PBS was injected into the IP cavity, and the abdomen was massaged to recover the inflammatory cells adherent to the abdominal organs and peritoneal cavity. The peritoneal exudate was collected in a syringe and stored on ice until further analysis. The total number of leukocytes was counted using a hemocytometer, and cytospins of $1 \times 10^{6}$ cells were performed by cytocentrifugation ( $5 \mathrm{~min}$ at $1000 \mathrm{rpm}$ ). Cells were then gently washed in tap water and fixed in formaldehyde-ethanol (50/50 [v/v] formaldehyde $3.7 \%$ and absolute ethanol) for $45 \mathrm{~s}$. The Wright's staining for blood samples (Hemacolor) was performed, and after being dried, each slide was observed in an optical microscope. A minimum of 300 cells per sample were counted in different areas of the cytospin and the different types of leukocytes distinguished.

\section{Histological preparations}

The animals with the implanted SI-M, dSI-M, and Cht/ Soy-M were anesthetized, at the end of each implantation period, respectively, with an IP injection of $2.5 \%$ pentobarbital or with an intramuscular injection of $90 \mathrm{mg} / \mathrm{kg}$ ketamine hydrochloride and $5 \mathrm{mg} / \mathrm{kg}$ xylazine hydrochloride, and sacrificed with an intracardial overdose of anesthetic. The implanted membranes, the respective surrounding tissue, and the lymph nodes of the rats with Cht/Soy-M were explanted and prepared for histological analysis using hematoxylin and eosin staining and subsequently analyzed using an Axioplan Imager Z1 microscope (Zeiss). Inflammatory reaction, integration of the membranes in the host tissue, and in vivo degradation were the assessed parameters.

\section{Statistical analysis}

Mean values and standard deviations are reported for the measurements of the leukocyte kinetics. Data were analyzed by a single-factor analysis of variance test and the significance value was set at $p<0.05$.

\section{Results \\ Leukocyte recruitment kinetics}

The IP injection of a suspension of SI-P allowed creating, after counting the existing subpopulations, the kinetic of leukocyte recruitment into in the IP cavity subsequent to its injection.

The number of PMNs, expected to be the first recruited cell type, was negligible in the negative control, showing that the injection of the saline did not elicit a significant recruitment of those cells. Few PMNs were found at $16 \mathrm{~h}$ after the saline injection, which is expected as a normal reaction to the injection.

Conversely, PMNs were extensively recruited early after injection of the SI-P suspensions. The number of recruited PMNs reached a peak $16 \mathrm{~h}$ postinjection of the $0.1 \%$ SI-P suspension and decreased to values similar to the control from that time point onward. After injection of the higher concentration of SI-P (1\%), higher numbers of PMNs were detected up to 3 days while consecutively decreasing.

Concerning the number of PMNs after the injection of the Cht suspensions, a recruitment pattern similar to the negative control was observed at all times. A higher, but not statistically significant $(p>0.05)$, number of PMNs was recruited in the presence of the higher concentration of Cht-P $(2 \%) 16 \mathrm{~h}$ postinjection. This number decreased afterward to comparable values to the observed for lower concentrations of Cht-P and for the control. 
Macrophages (MØ), either tissue resident or recruited from the bloodstream, are the most abundant cells in the IP cavity at physiological conditions. Therefore, the constant number of MØ observed for the control along the time was expected and attributed to resident cells. Sixteen hours after the injection of the SI-P, the number of detected MØ was higher than that for the negative control $(p<0.05)$ and associated, together with the PMNs reaction, to an expected inflammatory reaction to any implantation procedure. MØ recruitment reached a maximum between days 3 and $7(p<0.05)$, and between 7 and 14 days $(p<0.05)$, respectively for $0.1 \%$ and $1 \%$ of SI-P. The number of MØs started to decrease from that day onward although a significantly higher number, compared to the negative control, was detected at day 15 for $1 \%$ SI-P.

Regarding the number of $\mathrm{M} \varnothing$ present in the peritoneal cavity of the animals injected with the three different concentrations of Cht-P, no significant differences $(p>0.05)$ were observed between them and in comparison to the negative control at all time points.

In the context of a chronic inflammation, it is expected that lymphocytes, the type of cells that correlate with the onset of the reaction, would start to migrate from circulation around day 7 after the inflammatory challenge. Nonetheless, lymphocytes are also resident cells in the peritoneal cavity. The presence of lymphocytes was in fact noticed in the negative control at earlier time points. An increase $(p<0.05)$ in the number of lymphocytes was observed after the injection of the SI-P. The percentage of SI-P and the time of reaction did not induce significant changes over the recruitment kinetics except for $0.1 \%$ SI-P at day 3 that presented a value significantly different from the control. As for the PMNs and MØ, the number of recruited lymphocytes to the peritoneal cavity in the animals injected with the three different concentrations of Cht$\mathrm{P}$ was comparable to the negative control at all time points.

The recruitment of eosinophils and mast cells, which are mostly related with allergic reactions, was negligible after the injection of the saline (negative control). The same profile was observed for all of the concentrations of Cht-P and SI-P during the observation time. An exception was detected for the $0.1 \%$ SI-P solution, which induced a maximum of eosinophil and mast cell exudation at day 3 , although the increase was not statistically significant $(p>0.05)$. From that time period onward the number of these cells decreased, reaching levels comparable to the other tested concentration and the negative control.

\section{Histological analysis}

I. SI-M and dSI-M degradation and tissue inflammatory response. Animals did not show any surgical complications during the postoperative period. The explantation of SI-M, $\mathrm{dSI}-\mathrm{M}$, and the respective surrounding tissue was performed at $3,7,14$, and 30 days after the subcutaneous implantation and processed for histological analysis. Inflammatory reaction, integration of the membranes in the host tissue, and in vivo degradation were the assessed parameters.

The obtained histological sections revealed that, after 3 days of implantation, the SI-M maintained its integrity (Fig. 1A, B) compared to the dSI-M (Fig. 1C, D), which lost some of its integrity. Additionally, the extension of the observed inflammatory infiltrate was higher in the dSI-M (Fig. 1C, D) than in the SI-M (Fig. 1A, B), demonstrating that the dSI-M elicited higher recruitment of inflammatory cells in comparison to the SI-M. The histological examination at day 3 of implantation revealed the physical separation between the membranes and the adjacent tissue, resulting from the processing procedure. However, it was possible to notice a more evident detachment of the SI-M (Fig. 1A) from the inflammatory infiltrate in contrast to the dSI-M (Fig. 1C) in which attached inflammatory cells were easily identified. A detailed observation of the histological sections showed that the inflammatory infiltrate is mainly constituted of PMNs, characterized by multilobulated nuclei, recruited from circulation in response to either the SI-M (Fig. 1B) or the dSI-M (Fig. 1D). Nonetheless, some mononuclear cells, presenting a smaller cytoplasm and a round shaped nucleus, were also observed in both situations.

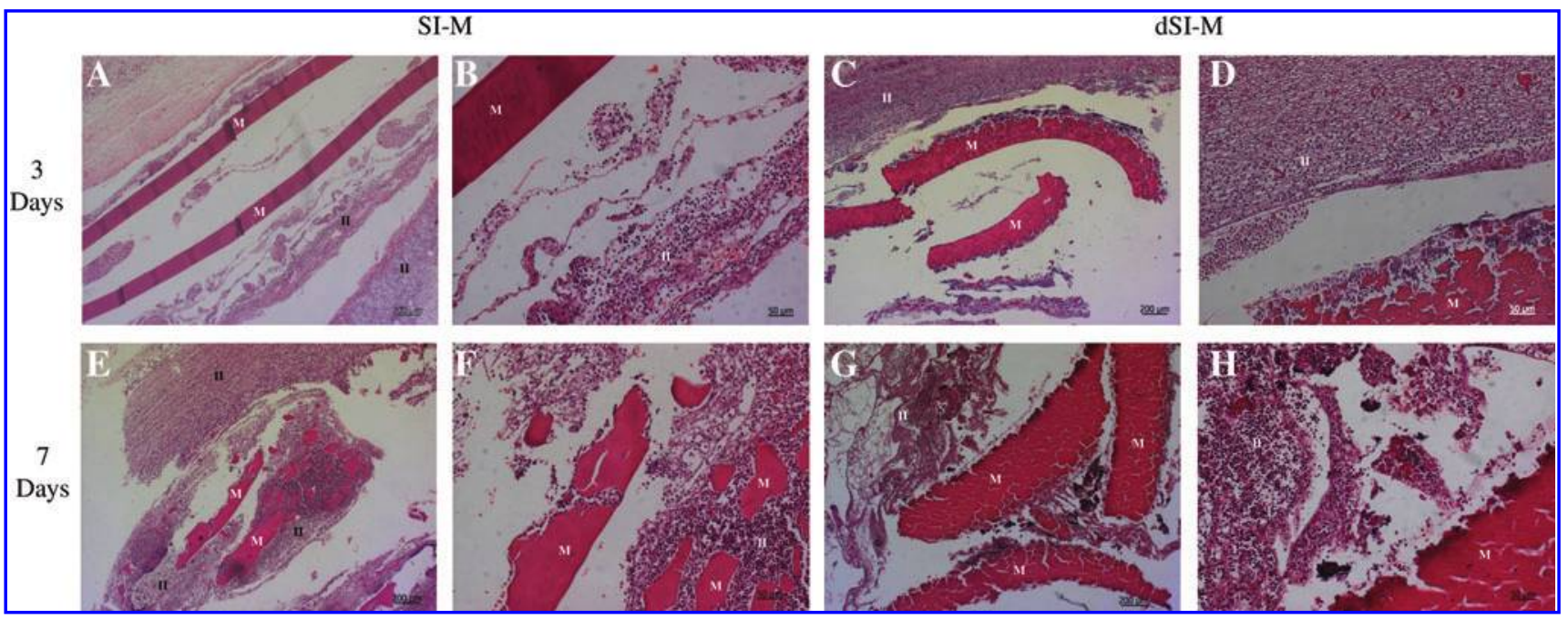

FIG. 1. Microscopic images obtained from the explanted SI-M (A, B, E, F) and dSI-M (C, D, G, H) and surrounding tissue, subcutaneously implanted for $3(\mathbf{A}, \mathbf{B}, \mathbf{C}, \mathbf{D})$ and $7(\mathbf{E}, \mathbf{F}, \mathbf{G}, \mathbf{H})$ days in rats. It is possible to observe the membrane (M) surrounded by inflammatory infiltrate (II). SI-M, soybean protein isolate membrane; dSI-M, denatured SI-M. Color images available online at www.liebertonline.com/ten. 


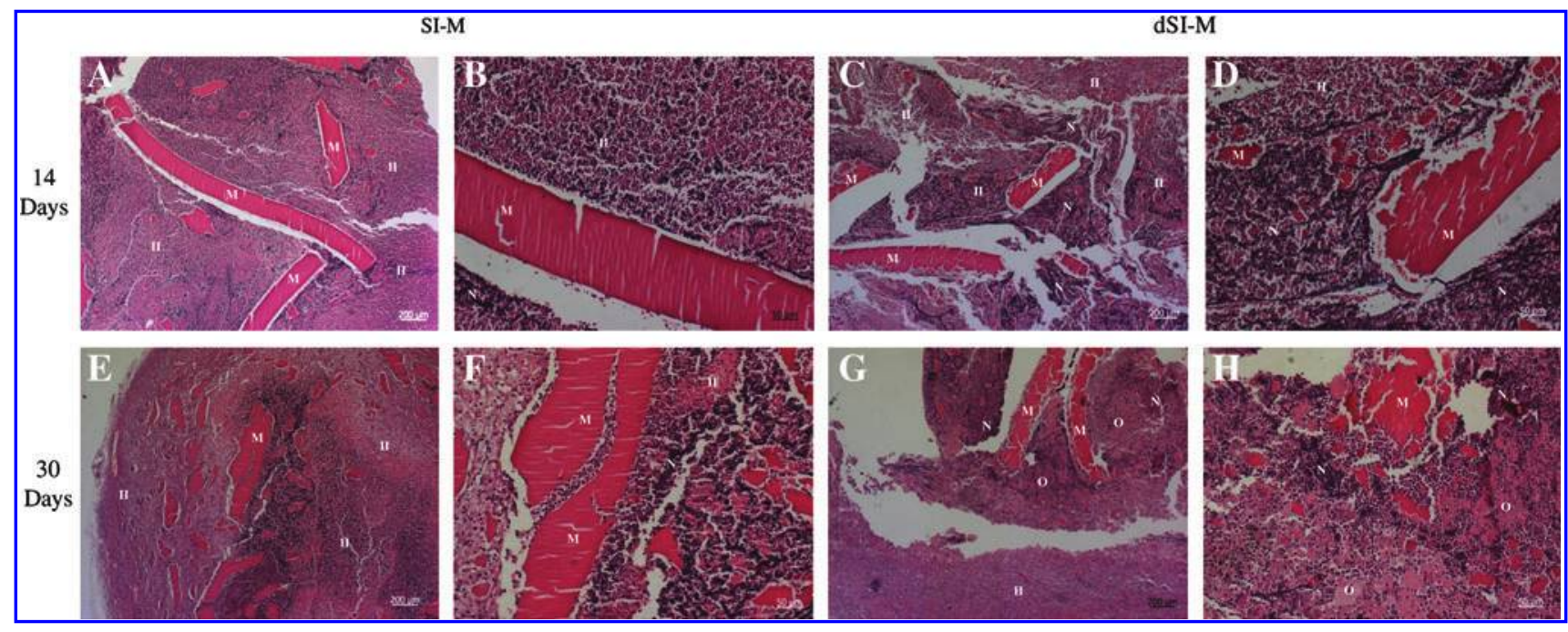

FIG. 2. Microscopic images obtained from the explanted SI-M (A, B, E, F) and dSI-M (C, D, G, H) and surrounding tissue, subcutaneously implanted for $14(\mathbf{A}, \mathbf{B}, \mathbf{C}, \mathbf{D})$ and $30(\mathbf{E}, \mathbf{F}, \mathbf{G}, \mathbf{H})$ days in rats. At these time points, there is a denser inflammatory infiltrate (II) surrounding the membrane (M), and some necrosis $(\mathrm{N})$ and edema $(\mathrm{O})$ is observed. Color images available online at www.liebertonline.com/ten.

After 7 days of the subcutaneous implantation of the SI-M and the dSI-M, the density of the inflammatory infiltrate increased (Fig. 1E, G). The degradation of both types of membranes was obvious after 7 days of implantation (Fig. $1 \mathrm{E}-\mathrm{H})$, although more noticeable, like at 3 days of implantation, for the dSI-M. Moreover, a network of fibrotic tissue was observable surrounding the inflammatory infiltrate, again characterized by the presence of PMNs, which seem to be attempting to phagocyte the polymeric material, and mononuclear cells. In the tissue surrounding the dSI-M, the inflammatory infiltrate was denser, and early signs of edema formation and necrosis with the exteriorization of cell cytoplasm, and the presence of cell debris and picnotic nucleus were clearly identified (Fig. 1H).

After 14 days of implantation, the expected resolution of a normal inflammatory process was not occurring. In the case of the SI-M, the PMNs persisted (Fig. 2A, B). Additionally, cell apoptosis and necrosis are evident (Fig. 2B) and seem to increase in comparison to 7 days of implantation. These observations were even more evident for the dSI-M; the density of the inflammatory infiltrate was higher and the signs of necrosis were even clearer (Fig. 2C, D). At this stage, the degradation of the dSI-M was also more pronounced, compared to both the SI-M and the first 7 days of implantation of the dSI-M.

After 30 days of subcutaneous implantation of the SI-M and dSI-M, the degradation of the membranes, the edema, and necrosis evidently increased more compared with the previous implantation periods (Fig. 2E-H).

II. Cht/Soy-M degradation and tissue inflammatory response. The Cht/Soy-Ms were subcutaneously implanted in rats for 7 and 14 days. During the postimplantation period no signals of systemic or regional surgical complications were detected for any of the animals. The local and systemic host responses to the implanted materials were analyzed after histological processing of the explants comprising the implanted Cht/Soy-M and respective surrounding tissue, and of the axillary and inguinal lymph nodes.
Local reaction to the Cht/Soy-M after 7 days of implantation comprised an inflammatory infiltrate formed by recruited PMNs (Fig. 3A). Additionally, some matrix started to be deposited around resident and inflammatory cells present in the subcutaneous tissue. Further, blood vessels and adipocytes were also observed in the surroundings of the implantation site (Fig. 3B). Concerning the systemic host response, the analysis of the neighboring lymph nodes revealed its reactive state characterized by a higher cell density of the germinal centers compared to the medullar region (Fig. 3C). Additionally, no material debris was observed into the lymph nodes (Fig. 3C).

After 14 days of implantation, the progress of the inflammatory reaction was noticed; the inflammatory infiltrate was concentrated a few microns from the membrane, and the newly developed vascularization of connective tissue was evident (Fig. 3D, E). The presence of mononuclear cells that typically appear 2 weeks after the implantation of the material, and the absence of persistent PMNs were detected. Moreover, foreign body giant cells, one of the typical features of chronic inflammation, were not observed at the materialtissue interface (Fig. 3E). Similar to what was observed for day 7 of implantation, the neighboring lymph nodes showed comparable signs of reactivity (Fig. 3F).

\section{Discussion}

The combination of one of the most used natural materials, Cht, 6,13,14,36 with a rather promising one, soy, ${ }^{5,37}$ has recently been shown to possess very interesting features $7,8,31,33$ that should be explored within the context of potential biomedical applications. Following these recent findings and considering that there is a significant number of reports in the literature that highlight soy's inflammatory and allergic character, 11,38 the evaluation of the in vivo behavior of those materials was missing. Therefore, this study aimed to investigate the in vivo host reaction of these materials.

One of the most relevant features of biodegradable biomaterials that determine a successful performance refers to its degradation rate and subsequent degradation products. 


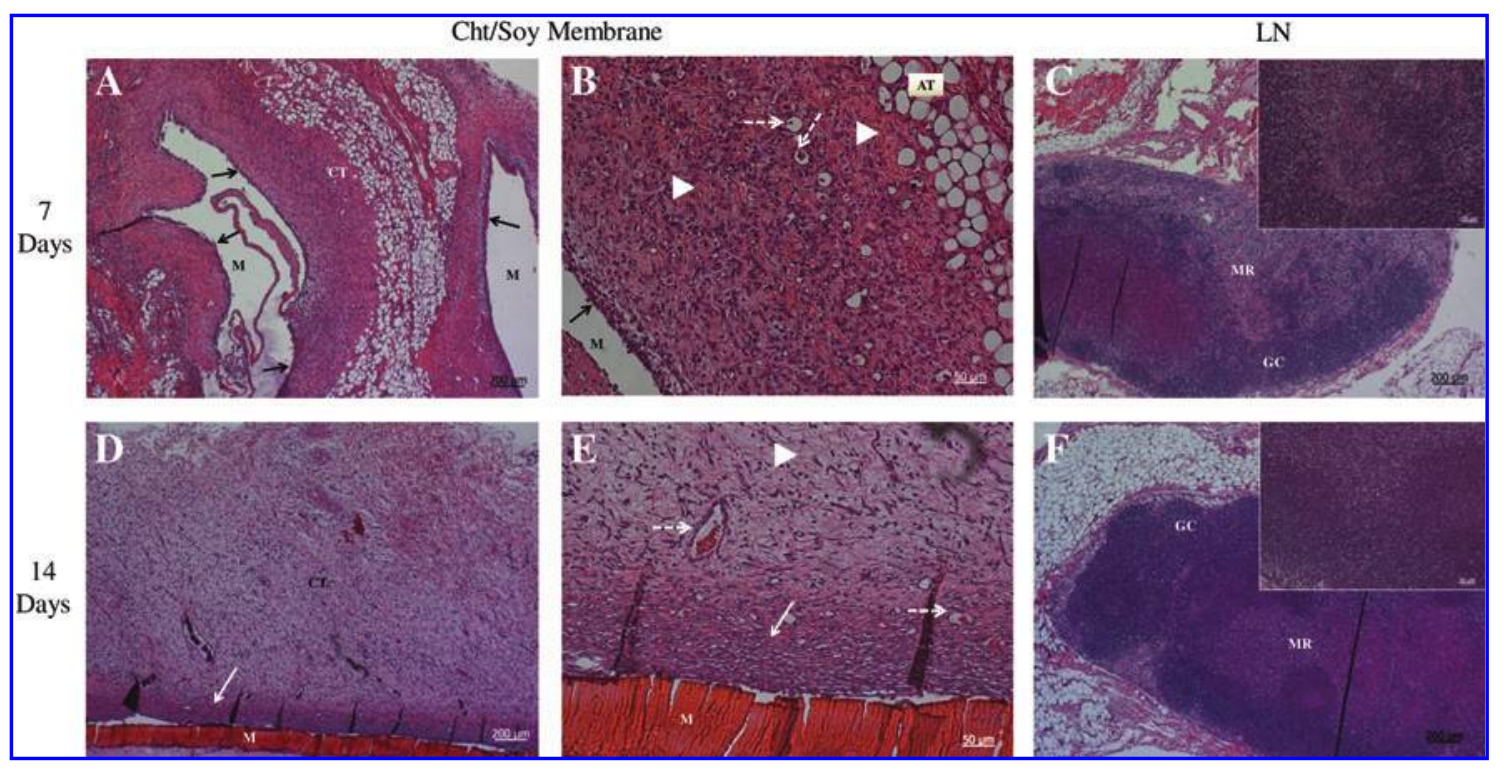

FIG. 3. Microscopic images obtained from the explanted Cht/soy-based membranes and surrounding tissue, subcutaneously implanted for $7(\mathbf{A}, \mathbf{B})$ and 14 days $(\mathbf{D}, \mathbf{E})$ in rats, as well as the nearby LN $(\mathbf{C}, \mathbf{F})$. Solid arrows $(\rightarrow)$ indicate inflammatory cells; broken arrows (-- $\rightarrow$ ), blood vessels; arrowheads: collagen fibers. M, membrane; AT, adipose tissue; CT, connective tissue; GC, germinal center; MR, medullar region; Cht, chitosan; LN, lymph nodes. The inserts in figures $\mathbf{C}$ and $\mathbf{F}$ represent higher magnification images of the medular region and germinal centers. Color images available online at www.liebertonline.com/ten.

The direct reaction to these as well as the inability of the host to deal with those products has been frequently reported as the major cause of implant failure. ${ }^{39}$ Thus, the testing of Cht and soy materials was considered relevant for a first assessment of the inflammatory and allergic potential of the materials in study. The host tissue response to both Cht and soy suspensions was monitored by investigating the IP leukocyte kinetics at four different periods $(3,7,14$, and 30 days). The basal level of reaction, induced by the procedure, was established with the injection of a saline and showed the typical population of inflammatory cells present in the rat peritoneal cavity in a physiological condition of minor trauma due to injection. ${ }^{40} \mathrm{MØs}$, the IP-tissue-resident cells, were the most evident type of cells detected. Although not at significant numbers, lymphocytes can also be present in the tissues, ${ }^{41}$ which justify the obtained values for the control.

Concerning leukocyte recruitment by the injection of SI and Cht as suspensions from the powders into the IP cavity of rats, SI-P elicited the recruitment of higher cell numbers compared to Cht-P at all times studied. In this sense, SI-P was considered more reactive to the host comparing to the Cht-P, which, independently of the concentration, elicited leukocyte recruitment comparable to the negative control. Although it would be expected that a direct effect exists that increasing the concentration of the suspension results in an increase in the intensity of the provoked reaction, this was not observed for the Cht-P, which is in agreement with previous studies with Cht-based materials, showing a typical inflammatory reaction induced by its implantation. ${ }^{42}$

The observed host response also did not seem to directly correlate with the different concentrations of the tested SI-P. SI induced a persistent recruitment of all inflammatory cell types in comparison with the Cht-P and negative control, since mononuclear cells ( $\mathrm{M \varnothing}$ and lymphocytes), the hallmark of a chronic inflammation, were extensively present at the latter stage of the reaction (14 days). These results seem to be in accordance with the observed reaction after the subcutaneous implantation of the membranes. Despite the absence of physiologic signs of inflammation or infection, the histological analysis of the explants revealed a severe host inflammatory reaction. Comparing the SI-M and the dSI-M, it was observed that the reaction to dSI-M was more intense. The extension of the inflammatory infiltrate was representative of an acute persistent reaction characterized by the presence of PMNs at longer implantation periods (14 and 30 days) that undergo frustrated phagocytosis. The higher degradation rate of the dSI-M, in comparison to SI-M and subsequent presence of smaller fragments of the membrane, might be responsible for the stronger reaction since a higher surface area is available for PMNs to respond to. The metabolites secreted by the PMNs in this situation lead to the decrease in the physiological $\mathrm{pH}$ and to the apoptosis and necrosis of neighboring cells, evident at later stages of implantation. Other studies ${ }^{43-45}$ suggested that surface properties such as hydrophilicity and surface charge determine inflammatory cells apoptosis and necrosis. Nevertheless, a typical foreign body reaction characterized by the presence of foreign body giant cells able to phagocytize large material debris $(>10 \mu \mathrm{m})^{46}$ was not observed. This seems to indicate that the degradation products of the implanted material are either $<10 \mu \mathrm{m}$ and thus phagocytozed by $\mathrm{M \varnothing}$, or metabolically re-absorbed by the host. These findings, showing the absence of foreign body giant cells in the host tissue after the implantation of Cht/Soy-M, are not typical for biodegradable polymeric materials whose degradation products lead to $\mathrm{MØ}$ fusion and fibrous tissue formation. ${ }^{47}$

The results of the leukocyte kinetics, where the SI-P induced a severe and persistent recruitment of inflammatory cells in the IP cavity of the rats after injection, were indicative of the expected subcutaneous response and could be ex- 
trapolated from previous reports of inflammation caused by soy products. ${ }^{11,38}$

The addition of Cht to the Soy-M improved, as expected, the host response, which showed the features of a typical inflammatory response to implanted materials. ${ }^{19-21,24}$ Moreover, the integration of the membranes within the surrounding tissue was revealed by the presence of matrix $^{39}$ after 2 weeks of implantation. These results are in agreement with the results of other researchers, reporting the in vitro inhibition of anti-inflammatory cytokines by Cht, such as interleukin-6, interleukin-8, and tumor necrosis factor- $\alpha$, ${ }^{48,49}$ which tend to prolong the acute inflammatory response, instead of allowing the host to progress to the resolution of inflammation. ${ }^{46}$ The nonactivated state of the lymph nodes after Cht/Soy-M implantation indicates that the implanted materials were not able to induce a more systemic host reaction and no debris or molecules of the materials were transported through circulation to induce a remote response of the related lymphatic tissues.

The presented results assert the improvement of the host response, considering inflammatory cells recruitment and overall of the inflammatory reaction, when Cht is added to soybean. In the context of previous indications on the behavior of PMNs after stimulation with Cht/Soy-M, ${ }^{31}$ the present results may assert the influence of Cht on masking specific soy reactive epitopes or even on suppressing leukocyte activation, namely, PMNs. The Cht/Soy-M showed the induction of a normal inflammatory reaction, and the features characterizing this reaction are crucial for the integration of the implanted material, as well as for the ongoing process of wound healing and tissue regeneration. Together with previous results that reported their promising physicochemical characteristics and their inability to activate human PMNs in vitro, ${ }^{31}$ the herein presented conclusions reinforce the usefulness of the Cht/Soy-M and justify the pursuit for a specific application within the biomedical field.

\section{Acknowledgments}

The author Tírcia C. Santos acknowledges the Marie Curie European Program for a short-term scholarship in the Alea Jacta EST project (MEST-CT-2004-008104). This work was developed under the scope of the European Network of Excellence EXPERTISSUES (NMP3-CT-2004-5000283).

\section{Disclosure Statement}

No competing financial interests exist.

\section{References}

1. Vaz, C.M., De Graaf, L.A., Reis, R.L., and Cunha, A.M. Effect of crosslinking, thermal treatment and UV irradiation on the mechanical properties and in vitro degradation behavior of several natural proteins aimed to be used in the biomedical field. J Mater Sci Mater Med 14, 789, 2003.

2. Vaz, C.M., Fossen, M., van Tuil, R.F., de Graaf, L.A., Reis, R.L., and Cunha, A.M. Casein and soybean protein-based thermoplastics and composites as alternative biodegradable polymers for biomedical applications. J Biomed Mater Res A 65, 60, 2003.

3. Vaz, C.M., van Doeveren, P.F., Dias, G.R., Reis, R.L., and Cunha, A.M. Controlled delivery achieved with bi-layer matrix devices produced by co-injection moulding. Macromol Biosci 4, 795, 2004.

4. Vaz, C.M., van Doeveren, P.F., Reis, R.L., and Cunha, A.M. Soy matrix drug delivery systems obtained by melt-processing techniques. Biomacromolecules 4, 1520, 2003.

5. Silva, G.A., Vaz, C.M., Coutinho, O.P., Cunha, A.M., and Reis, R.L. In vitro degradation and cytocompatibility evaluation of novel soy and sodium caseinate-based membrane biomaterials. J Mater Sci Mater Med 14, 1055, 2003.

6. Silva, R.M., Silva, G.A., Coutinho, O.P., Mano, J.F., and Reis, R.L. Preparation and characterisation in simulated body conditions of glutaraldehyde crosslinked chitosan membranes. J Mater Sci Mater Med 15, 1105, 2004.

7. Silva, S.S., Santos, M.I., Coutinho, O.P., Mano, J.F., and Reis, R.L. Physical properties and biocompatibility of chitosan/ soy blended membranes. J Mater Sci Mater Med 16, 575, 2005.

8. Silva, S.S., Oliveira, J.M., Mano, J.F., and Reis, R.L. Physicochemical characterization of novel chitosan-soy protein/ TEOS porous hybrids for tissue engineering applications. Materials Sci Forum 514, 1000, 2006.

9. Santin, M., Morris, C., Standen, G., Nicolais, L., and Arnbrosio, L. A new class of bioactive and biodegradable soybeanbased bone fillers. Biomacromolecules 8, 2706, 2007.

10. L'Hocine, L., and Boye, J.I. Allergenicity of soybean: new developments in identification of allergenic proteins, crossreactivities and hypoallergenization technologies. Crit Rev Food Sci Nutr 47, 127, 2007.

11. Mittag, D., Vieths, S., Vogel, L., Becker, W.M., Rihs, H.P., Helbling, A., Wuthrich, B., and Ballmer-Weber, B.K. Soybean allergy in patients allergic to birch pollen: clinical investigation and molecular characterization of allergens. J Allergy Clin Immunol 113, 148, 2004.

12. Tuzlakoglu, K., Alves, C.M., Mano, J.F., and Reis, R.L. Production and characterization of chitosan fibers and 3-D fiber mesh scaffolds for tissue engineering applications. Macromol Biosci 4, 811, 2004.

13. Kim, I.Y., Seo, S.J., Moon, H.S., Yoo, M.K., Park, I.Y., Kim, B.C., and Cho, C.S. Chitosan and its derivatives for tissue engineering applications. Biotechnol Adv 26, 1, 2008.

14. Di Martino, A., Sittinger, M., and Risbud, M.V. Chitosan: a versatile biopolymer for orthopaedic tissue-engineering. Biomaterials 26, 5983, 2005.

15. Zhang, Y., Ni, M., Zhang, M., and Ratner, B. Calcium phosphate-chitosan composite scaffolds for bone tissue engineering. Tissue Eng 9, 337, 2003.

16. Wu, H., Wan, Y., Dalai, S., and Zhang, R. Response of rat osteoblasts to polycaprolactone/chitosan blend porous scaffolds. J Biomed Mater Res A 92, 238, 2010.

17. Lu, J.X., Prudhommeaux, F., Meunier, A., Sedel, L., and Guillemin, G. Effects of chitosan on rat knee cartilages. Biomaterials 20, 1937, 1999.

18. Yamane, S., Iwasaki, N., Majima, T., Funakoshi, T., Masuko, T., Harada, K., Minami, A., Monde, K., and Nishimura, S. Feasibility of chitosan-based hyaluronic acid hybrid biomaterial for a novel scaffold in cartilage tissue engineering. Biomaterials 26, 611, 2005.

19. Ueno, H., Yamada, H., Tanaka, I., Kaba, N., Matsuura, M., Okumura, M., Kadosawa, T., and Fujinaga, T. Accelerating effects of chitosan for healing at early phase of experimental open wound in dogs. Biomaterials 20, 1407, 1999.

20. Cho, Y.W., Cho, Y.N., Chung, S.H., Yoo, G., and Ko, S.W. Water-soluble chitin as a wound healing accelerator. Biomaterials 20, 2139, 1999. 
21. Peluso, G., Petillo, O., Ranieri, M., Santin, M., Ambrosio, L., Calabro, D., Avallone, B., and Balsamo, G. Chitosan-mediated stimulation of macrophage function. Biomaterials 15, 1215, 1994.

22. Ueno, H., Nakamura, F., Murakami, M., Okumura, M., Kadosawa, T., and Fujinag, T. Evaluation effects of chitosan for the extracellular matrix production by fibroblasts and the growth factors production by macrophages. Biomaterials 22, 2125, 2001.

23. Rucker, M., Laschke, M.W., Junker, D., Carvalho, C., Schramm, A., Mulhaupt, R., Gellrich, N.C., and Menger, M.D. Angiogenic and inflammatory response to biodegradable scaffolds in dorsal skinfold chambers of mice. Biomaterials 27, 5027, 2006.

24. Azab, A.K., Doviner, V., Orkin, B., Kleinstern, J., Srebnik, M., Nissan, A., and Rubinstein, A. Biocompatibility evaluation of crosslinked chitosan hydrogels after subcutaneous and intraperitoneal implantation in the rat. J Biomed Mater Res A 83, 414, 2007.

25. Hong, Y., Mao, Z., Wang, H., Gao, C., and Shen, J. Covalently crosslinked chitosan hydrogel formed at neutral $\mathrm{pH}$ and body temperature. J Biomed Mater Res A 79, 913, 2006.

26. Cai, K., Hu, Y., Jandt, K.D., and Wang, Y. Surface modification of titanium thin film with chitosan via electrostatic self-assembly technique and its influence on osteoblast growth behavior. J Mater Sci Mater Med 19, 499, 2008.

27. Bumgardner, J.D., Wiser, R., Gerard, P.D., Bergin, P., Chestnutt, B., Marin, M., Ramsey, V., Elder, S.H., and Gilbert, J.A. Chitosan: potential use as a bioactive coating for orthopaedic and craniofacial/dental implants. J Biomater Sci Polym Ed 14, 423, 2003.

28. Lee, J.Y., Nam, S.H., Im, S.Y., Park, Y.J., Lee, Y.M., Seol, Y.J., Chung, C.P., and Lee, S.J. Enhanced bone formation by controlled growth factor delivery from chitosan-based biomaterials. J Controlled Release 78, 187, 2002.

29. Bumgardner, J.D., Chesnutt, B.M., Yuan, Y., Yang, Y., Appleford, M., Oh, S., McLaughlin, R., Elder, S.H., and Ong, J.L. The integration of chitosan-coated titanium in bone: an in vivo study in rabbits. Implant Dent 16, 66, 2007.

30. Coutinho, D.F., Pashkuleva, I.H., Alves, C.M., Marques, A.P., Neves, N.M., and Reis, R.L. The effect of chitosan on the in vitro biological performance of chitosan-poly(butylene succinate) blends. Biomacromolecules 9, 1139, 2008.

31. Santos, T.C., Marques, A.P., Silva, S.S., Oliveira, J.M., Mano, J.F., Castro, A.G., and Reis, R.L. In vitro evaluation of the behaviour of human polymorphonuclear neutrophils in direct contact with chitosan-based membranes. J Biotechnol 132, 218, 2007.

32. Signini, R., and Filho, S.P.C. On the preparation and characterization of chitosan hydrochloride. Polym Bull 42, 159, 1999.

33. Silva, R.M., Elvira, C., Mano, J.F., San Roman, J., and Reis, R.L. Influence of beta-radiation sterilisation in properties of new chitosan/soybean protein isolate membranes for guided bone regeneration. J Mater Sci Mater Med 15, 523, 2004.

34. Larena, A., and Caceres, D.A. Variability between chitosan membrane surface characteristics as function of its composition and environmental conditions. Appl Surface Sci 238, 273, 2004.

35. Rhim, J.W., Gennadios, A., Handa, A., Weller, C.L., and Hanna, M.A. Solubility, tensile, and color properties of modified soy protein isolate films. J Agric Food Chem 48, 4937, 2000.

36. Shi, C., Zhu, Y., Ran, X., Wang, M., Su, Y., and Cheng, T. Therapeutic potential of chitosan and its derivatives in regenerative medicine. J Surg Res 133, 185, 2006.
37. Santin, M., and Ambrosio, L. Soybean-based biomaterials: preparation, properties and tissue regeneration potential. Expert Rev Med Devices 5, 349, 2008.

38. Steinman, H.A. "Hidden" allergens in foods. J Allergy Clin Immunol 98, 241, 1996.

39. Williams, D.F. On the mechanisms of biocompatibility. Biomaterials 29, 2941, 2008.

40. Goldsby, R.A., Kindt, T.J., and Osborne, B.A. Kuby Immunology. W.H. Freeman and Company, 2000.

41. Rajakariar, R., Lawrence, T., Bystrom, J., Hilliard, M., Colville-Nash, P., Bellingan, G., Fitzgerald, D., Yaqoob, M.M., and Gilroy, D.W. Novel biphasic role for lymphocytes revealed during resolving inflammation. Blood 111, 4184, 2008.

42. Malafaya, P.B., Santos, T.C., van Griensven, M., and Reis, R.L. Morphology, mechanical characterization and in vivo neo-vascularization of chitosan particle aggregated scaffolds architectures. Biomaterials 29, 3914, 2008.

43. Peluso, G., Petillo, O., Ranieri, M., Santin, M., Ambrosio, L., Calabro, D., Avallone, B., and Balsamo, G. Chitosan-mediated stimulation of macrophage function. Biomaterials 15, 1215, 1994.

44. Brodbeck, W.G., Patel, J., Voskerician, G., Christenson, E., Shive, M.S., Nakayama, Y., Matsuda, T., Ziats, N.P., and Anderson, J.M. Biomaterial adherent macrophage apoptosis is increased by hydrophilic and anionic substrates in vivo. Proc Natl Acad Sci USA 99, 10287, 2002.

45. Mori, T., Murakami, M., Okumura, M., Kadosawa, T., Uede, T., and Fujinaga, T. Mechanism of macrophage activation by chitin derivatives. J Vet Med Sci 67, 51, 2005.

46. Anderson, J.M., Rodriguez, A., and Chang, D.T. Foreign body reaction to biomaterials. Semin Immunol 20, 86, 2008.

47. Yoon, S.J., Kim, S.H., Ha, H.J., Ko, Y.K., So, J.W., Kim, M.S., Yang, Y.I., Khang, G., Rhee, J.M., and Lee, H.B. Reduction of inflammatory reaction of poly(D,L-lactic-co-glycolic acid) using demineralized bone particles. Tissue Eng Part A 14, 539, 2008.

48. Kim, M.S., Sung, M.J., Seo, S.B., Yoo, S.J., Lim, W.K., and Kim, H.M. Water-soluble chitosan inhibits the production of pro-inflammatory cytokine in human astrocytoma cells activated by amyloid beta peptide and interleukin-1beta. Neurosci Lett 321, 105, 2002.

49. Kim, M.S., You, H.J., You, M.K., Kim, N.S., Shim, B.S., and Kim, H.M. Inhibitory effect of water-soluble chitosan on TNF-alpha and IL-8 secretion from HMC-1 cells. Immunopharmacol Immunotoxicol 26, 401, 2004.

Address correspondence to: Tírcia C. Santos, Ph.D.

3B's Research Group-Biomaterials, Biodegradables, and Biomimetics

University of Minho

Headquarters of the European Institute of Excellence on Tissue Engineering and Regenerative Medicine AvePark 4806-909 Taipas Guimarães Portugal

E-mail: tircia.santos@dep.uminho.pt

Received: February 23, 2010 Accepted: April 22, 2010

Online Publication Date: June 2, 2010 